\title{
Multimodal hybrid imaging agents for sentinel node mapping as a means to (re)connect nuclear medicine to advances made in robot-assisted surgery
}

\author{
Gijs H. KleinJan ${ }^{1,2}$ • Nynke S. van den Berg ${ }^{1,3}$ • Jeroen de Jong ${ }^{4}$ - Esther M. Wit ${ }^{3}$. \\ Helene Thygessen ${ }^{5}$ Erik Vegt $^{2} \cdot$ Henk G. van der Poel ${ }^{3} \cdot$ Fijs W. B. van Leeuwen ${ }^{1,3,6}$
}

Received: 8 October 2015 / Accepted: 10 December 2015 /Published online: 15 January 2016

(C) The Author(s) 2016. This article is published with open access at Springerlink.com

\begin{abstract}
Purpose Radical prostatectomy and complementary extended pelvic lymph node dissection (ePLND) of sentinel lymph nodes (SNs) and non-sentinel lymph nodes (LNs) at risk of containing metastases are increasingly being performed using high-tech robot-assisted approaches. Although this technological evolution has clear advantages, the physical nature of robotic systems limits the integrated use of routine radioguided surgery technologies. Hence, engineering effort in robotics are focused on the integration of fluorescence guidance technologies. Using the hybrid SN tracer indocyanine
\end{abstract}

Fijs W. B. van Leeuwen

F.W.B.van_Leeuwen@lumc.nl; http://

interventionalmolecularimaging.com

1 Interventional Molecular Imaging Laboratory, Department of Radiology, Leiden University Medical Hospital, Albinusdreef 2, 2300RC Leiden, The Netherlands

2 Department of Nuclear Medicine, The Netherlands Cancer InstituteAntoni van Leeuwenhoek Hospital, Plesmanlaan 121, 1066CX Amsterdam, The Netherlands

3 Department of Urology, The Netherlands Cancer Institute - Antoni van Leeuwenhoek Hospital, Plesmanlaan 121, 1066CX Amsterdam, The Netherlands

4 Department of Pathology, The Netherlands Cancer Institute - Antoni van Leeuwenhoek Hospital, Plesmanlaan 121, 1066CX Amsterdam, The Netherlands

5 Department of Biostatistics, The Netherlands Cancer Institute Antoni van Leeuwenhoek Hospital, Plesmanlaan 121, 1066CX Amsterdam, The Netherlands

6 Department of Head and Neck Surgery \& Oncology, The Netherlands Cancer Institute - Antoni van Leeuwenhoek Hospital, Plesmanlaan 121, 1066CX Amsterdam, The Netherlands green $-{ }^{99 \mathrm{~m}} \mathrm{Tc}$-nanocolloid (radioactive and fluorescent), for the first time in combination with a robot-integrated laparoscope, we investigated whether the robot-assisted approach affects the accuracy of fluorescence detection of SNs identified preoperatively using nuclear medicine.

Methods The study included 55 patients (Briganti nomogrambased risk $>5 \%$ on LN metastases) scheduled for robotassisted radical prostatectomy, SN biopsy and ePLND. Following indocyanine green- ${ }^{99 \mathrm{~m}} \mathrm{Tc}$-nanocolloid injection, preoperative nuclear imaging (lymphoscintigraphy and $\mathrm{SPECT} / \mathrm{CT}$ ) was used to locate the $\mathrm{SN}(\mathrm{s})$. The fluorescence laparoscope was used intraoperatively to identify the $\mathrm{SN}(\mathrm{s})$ with standard fluorescence settings (in 50 patients) and with customized settings (in 5 patients). The number and location of the SNs, the radioactive, fluorescence (both in vivo and ex vivo) and tumour status of the resected SNs/LNs, and postoperative complications were recorded and analysed.

Results Combined, preoperative lymphoscintigraphy and SPECT/CT imaging identified 212 SNs (median 4 per patient). Intraoperative fluorescence imaging using standard fluorescence settings visualized $80.4 \%(148 / 184 \mathrm{SNs} ; 50$ patients; ex vivo $97.8 \%$ ). This increased to $85.7 \%(12 / 14 \mathrm{SNs}$; 5 patients; ex vivo $100 \%$ ) with customized fluorescence settings. SPECT/CT images provided guidance towards the residual SNs. Ex vivo all removed SNs were radioactive. SNs were tumour-positive in $25.4 \%$ of patients (14/55; falsenegative rate $7 \%, 1 / 14$ patients). In ten patients, the $\mathrm{SN}$ was the only tumour-positive LN. Surgical complications were minimal.

Conclusion Directly linking 3D preoperative nuclear imaging information on SNs to a robot-integrated fluorescence laparoscope improved the surgeon's use of the technology and did not influence the sensitivity or morbidity of the procedure. To our surprise, however, the detection rates with the current fluorescence camera did not improve. 
Keywords Sentinel node $\cdot$ SPECT/CT $\cdot$ Prostate cancer . Fluorescence-guided surgery $\cdot$ Robot-assisted surgery

\section{Introduction}

In complex anatomies, accurate preoperative mapping of sentinel nodes (SNs) using nuclear medicine (lymphoscintigraphy with or without SPECT/CT) and intraoperative radioguidance are vital for planning and performing nodal resection $[1,2]$. Imaging in combination with the intraoperative use of gamma probes and/or portable gamma cameras has been shown to be valuable not only in patients with melanoma, penile, vulvar and breast cancer [3-5], but also during the sampling of pelvic SNs originating from prostate cancer [6-8]. With SPECT/CT it has become possible to accurately identify the anatomical location of SNs inside or outside the extended pelvic lymph node dissection (ePLND) template, and subsequently this information can be used for surgical planning.

In parallel with the technical evolution of nuclear medicine-based imaging, there has been a shift towards robot-assisted laparoscopic procedures in urology, and in particular for prostate cancer. Unfortunately, this shift has resulted in a mismatch between the two disciplines. Moreover, in robotic surgery, the urologist is no longer present alongside the patient, limiting the control he/she has on the use of imaging technologies without robot assistance such as the gamma probe [9]. At the same time, the robot arms physically restrict access for larger devices such as portable gamma cameras. On the positive side, new-generation laparoscopes can be equipped with an integrated fluorescence imaging option [10-12]. Hence, integration of this technology in the robotic workflow currently seems to be more straightforward. Fluorescence guidance towards SNs and non-sentinel lymph nodes (LNs) has been achieved in a robotic setting through the use of an additional fluorescence laparoscope and via a robotintegrated fluorescence laparoscope [9-11, 13]. Unfortunately, the fluorescent signal has very limited tissue penetration, meaning that more deeply lying $\mathrm{SNs} / \mathrm{LNs}$ may be missed when using fluorescence imaging alone [14]. Even worse, extensive surgery in the quest for a fluorescent signal may lead to an increase in surgical complications.

In 2009 , the hybrid tracer indocyanine green- ${ }^{99 \mathrm{~m}} \mathrm{Tc}$ nanocolloid (ICG- ${ }^{99 \mathrm{~m}}$ Tc-nanocolloid) was clinically introduced for SN biopsy. This tracer was designed to extend the European standard in nuclear medicine-based SN identification, ${ }^{99 \mathrm{~m}} \mathrm{Tc}$-nanocolloid, with intraoperative fluorescence guidance $[10,15-17]$. With this development, the strengths of radioguided procedures (e.g. a high sensitivity and indepth view of the SNs) are complemented by detailed realtime, but superficial, fluorescence guidance towards the preoperatively identified SNs. In combination with the introduction of an additional fluorescence laparoscope, the hybrid concept has provided a significant step forward in connecting preoperative lymphatic mapping and real-time intraoperative fluorescence-based SN identification [9]. However, the fact that the bedside assistant had to introduce and control the fluorescence laparoscope, rather than the operating urologist, was considered a limiting factor of this approach. We thus hypothesized that use of a robot-integrated fluorescence laparoscope could increase the level of control the operating urologist has, and could thus help increase the success rate in resecting preoperatively identified SNs via fluorescence guidance. To place these findings in perspective, the tumourpositive rate, sensitivity, false-negative rate and complication rate of SN biopsy using the this approach was evaluated and compared to SN biopsy in a historical cohort [9].

\section{Materials and methods}

\section{Patients}

Between January 2014 and July 2015, 50 patients with a Briganti nomogram-based risk of $>5 \%$ of LN metastases were included [18]. These patients were evaluated with the robot-integrated fluorescence laparoscope (standard settings). Between July 2015 and September 2015 another 5 patients were included for evaluation of the customized fluorescence imaging settings. All patients were scheduled for robot-assisted radical prostatectomy, ePLND and SN removal using the hybrid tracer, and provided written informed consent. The characteristics of the two groups of included patients are shown in Table 1.

\section{Preoperative SN mapping}

Tracer preparation, injection and preoperative imaging (lymphoscintigraphy and SPECT/CT) were performed as previously described by KleinJan et al. [9].

Briefly, the hybrid tracer ${ }^{99 \mathrm{~m}} \mathrm{Tc}$-nanocolloid was prepared by adding $2.0 \mathrm{~mL}$ pertechnetate (approximately $300 \mathrm{MBq}$ ) to a vial of nanocolloid (GE Healthcare, Eindhoven, The Netherlands). ICG $(0.05 \mathrm{~mL}, 5.0 \mathrm{mg} / \mathrm{mL}$ : PULSION Medical, Feldkirchen, Germany) was then added to the vial of ${ }^{99 \mathrm{~m}} \mathrm{Tc}$-nanocolloid. The total volume was subtracted from the volume of the vial and saline was added to reach a total volume of $2.0 \mathrm{~mL}$ in the syringe, after which the tracer was injected into the prostate under transrectal ultrasound guidance. The injection was followed by preoperative lymphoscintigraphy (15 min and $2 \mathrm{~h}$ after injection) and SPECT/CT imaging directly after acquisition of the late lymphoscintigram. After imaging acquisition the nuclear medicine physician determined the number and location of the SNs. 
Table 1 Patient characteristics

\begin{tabular}{|c|c|c|c|}
\hline & \multicolumn{2}{|l|}{ Group } & \multirow[t]{2}{*}{ Total } \\
\hline & $\mathrm{SN}+\mathrm{ePLND}$ & $\begin{array}{l}\mathrm{SN}+\mathrm{ePLND} \\
\text { customized settings }\end{array}$ & \\
\hline No. of patients & 50 & 5 & 55 \\
\hline Age (years), median (IQR) & $63(58-68)$ & $66(62-68)$ & $63(59-68)$ \\
\hline $\begin{array}{l}\text { Preoperative PSA level (ng/mL), } \\
\text { median (IQR) }\end{array}$ & $7.75(5.44-12.18)$ & $7.9(4.8-23.35)$ & $7.9(5.46-12)$ \\
\hline \multicolumn{4}{|l|}{ Clinical T stage, $n(\%)$} \\
\hline $1 \mathrm{c}$ & $6(12)$ & - & $6(11)$ \\
\hline $2 \mathrm{a}$ & $5(10)$ & - & $5(9)$ \\
\hline $2 b$ & $9(18)$ & $1(20)$ & $10(18)$ \\
\hline $2 \mathrm{c}$ & $14(28)$ & $1(20)$ & $15(27)$ \\
\hline $3 a$ & $12(24)$ & $2(40)$ & $14(25)$ \\
\hline $3 b$ & $4(8)$ & $1(20)$ & $5(9)$ \\
\hline \multicolumn{4}{|l|}{ Biopsy Gleason sum score, $n(\%)$} \\
\hline 6 & $3(6)$ & - & $3(5)$ \\
\hline 7 & $30(60)$ & $4(80)$ & $34(62)$ \\
\hline 8 & $15(30)$ & $1(20)$ & $16(29)$ \\
\hline 9 & $2(4)$ & - & $2(4)$ \\
\hline Briganti score, median (IQR) & $28(21-53)$ & $54(20-68)$ & $28(21-50)$ \\
\hline \multicolumn{4}{|l|}{ Clinical N stage, $n(\%)$} \\
\hline No & $44(88)$ & $5(100)$ & $49(89)$ \\
\hline $\mathrm{Nx}$ & $6(12)$ & - & $6(11)$ \\
\hline \multicolumn{4}{|l|}{ Pathological T stage, $n(\%)$} \\
\hline $2 \mathrm{a}$ & - & $1(20)$ & $1(2)$ \\
\hline $2 \mathrm{~b}$ & $3(6)$ & - & $3(5)$ \\
\hline $2 \mathrm{c}$ & $37(74)$ & $2(40)$ & $39(71)$ \\
\hline $3 a$ & $4(8)$ & $1(20)$ & $5(9)$ \\
\hline $3 b$ & $4(8)$ & $1(20)$ & $5(9)$ \\
\hline 4 & $1(2)$ & - & $1(2)$ \\
\hline $4 a$ & $1(2)$ & - & $1(2)$ \\
\hline \multicolumn{4}{|c|}{ Pathological Gleason sum score, $n(\%)$} \\
\hline 6 & $6(12)$ & $1(20)$ & 7 (13) \\
\hline 7 & $35(70)$ & $2(40)$ & 37 (67) \\
\hline 8 & 7 (14) & $1(20)$ & $8(14)$ \\
\hline 9 & $2(4)$ & $1(20)$ & $3(54)$ \\
\hline
\end{tabular}

\section{Surgical (imaging) tools}

For the surgical procedure a da Vinci Si system (Intuitive Surgical, Sunnyvale, CA, USA) with an integrated Firefly fluorescence laparoscope was used. Via the goggles of the master console, images obtained with the laparoscope are presented to the urologist. Using the controllers of the console the urologist is able to switch between the white light imaging mode and the fluorescence imaging mode of the fluorescence laparoscope. In the initial 50 patients fluorescence imaging with the standard settings was evaluated.

After a software upgrade (P9 software update) of the da Vinci Si system, the urologist was able to manually adjust the fluorescence illuminator settings. In this second set-up, with which 5 patients were evaluated, the intensity of the white light background in the fluorescence image was varied 
from $30 \%$ (standard settings) to $15 \%$ and $0 \%$. The visibility of the fluorescence signal within the SNs was evaluated at these three different settings.

\section{Surgical procedure}

Prior to the start of the operation, the operating urologist viewed the SPECT/CT images using a DICOM viewer (PACS Vue Solutions; Carestream Health, Rochester, NY). The SNs identified on SPECT were again related to their anatomical context using the anatomical information provided by the low-dose CT scan. This information was used to help guide the positioning of the surgical tools and the fluorescence laparoscope for optimal SN localization and resection. Preopeatively identified SNs in a location with a high risk of surgical complications were left in situ. The remaining SNs, after initial preparation of the area of interest, were optically identified by switching between white light imaging and fluorescence imaging (Fig. 1). After excision of the SN, the surgical bed was re-examined using a combination of white light and fluorescence imaging.

To simplify the surgical workflow, based on the results of our previous study [9], no laparoscopic gamma probe was used intraoperatively. However, ex vivo the gamma probe (Europrobe 2; Eurorad S.A., Eckbolsheim, France) was used to validate the radioactive signature in the excised $\mathrm{SNS} / \mathrm{LNs}$. To confirm the presence of fluorescence in the excised SNs, fluorescence imaging was performed ex vivo using a fluorescence camera for open surgery (PDE; Hamamatsu Photonics K.K., Hamamatsu, Japan).
The number and location of SNs identified preoperatively and intraoperatively were recorded together with their in vivo and ex vivo fluorescent and radioactive status.

Following SN excision, an ePLND was performed followed by the the radical prostatectomy. The ePLND included dissection of LNs from the following areas: around the external iliac artery and vein, within the obturator fossa, and surrounding the internal iliac artery [19].

\section{(Histo)pathological examination}

(Histo)pathological analysis of the SNs, additional LNs from the ePLND specimens and the prostate were performed as previously described [9].

\section{Follow-up}

Complications occurring within 90 days of the operation were recorded using the Clavien-Dindo classification and were evaluated in the initial 50 patients [20]. In the last 5 patients evaluated follow-up was not sufficiently long for them to be included in the postoperative evaluation. The findings were compared to those reported in our previous study in which we reported on fluorescence guidance without robotic integration [9].

\section{Statistical analysis}

Statistical analysis was performed using SPSS Statistics, version 22 (SPSS Inc., Chicago, IL). A $p$ - value $<0.05$ was

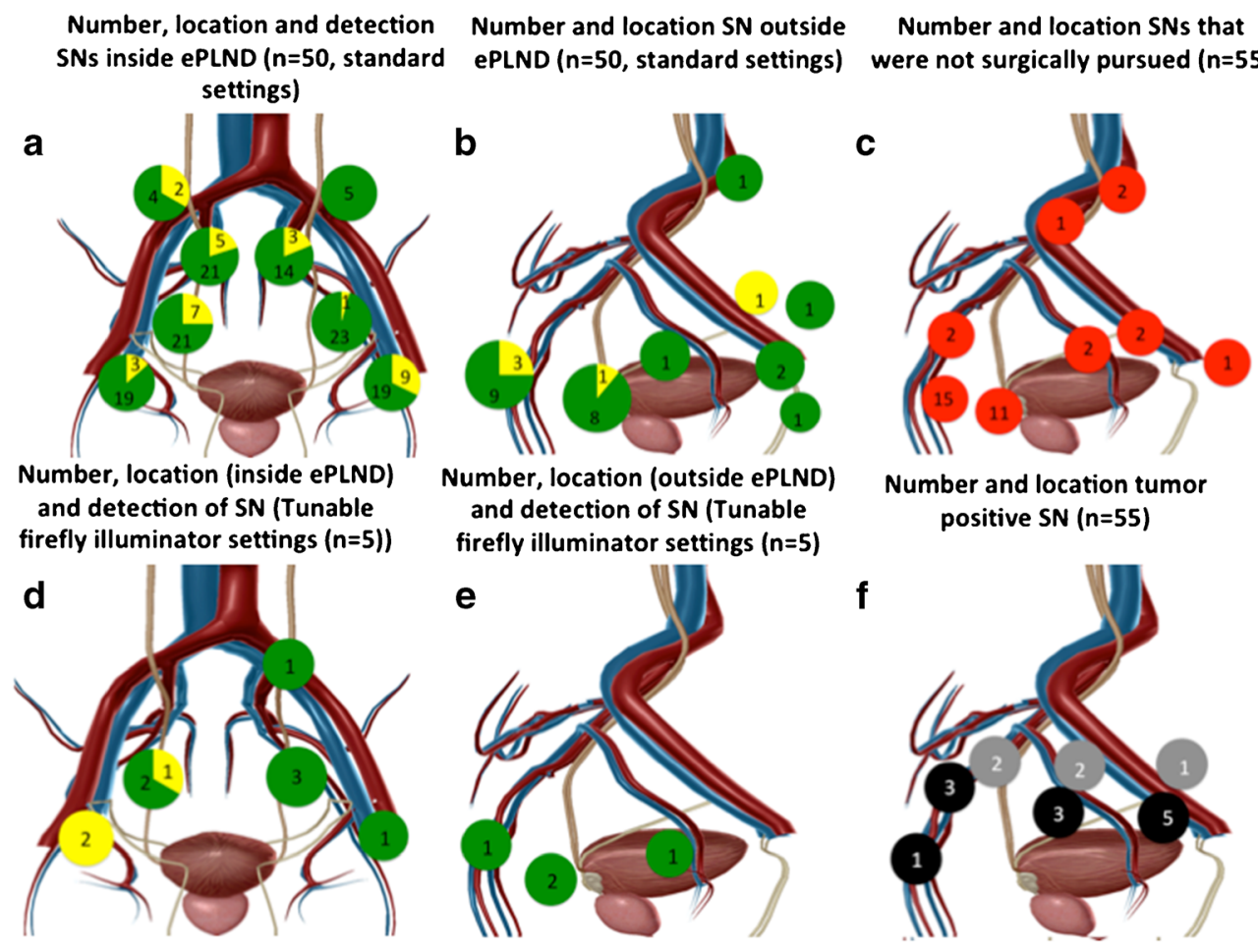

Fig. 1 Fluorescence-based SN identification: a white light image; b fluorescence-based image 
considered significant. For continuous variables, the mean or median and interquartile ranges (IQR) are presented. For discrete variables, frequencies and percentages are reported. The Welch two-sample $t$ - test was used to compare the preoperative Briganti scores, and the number of removed SNs and LNs in the patients in this study and in the patients in the previous study [9]. A Fisher's exact test was used to compare the tumour positivity rate ( $\mathrm{pN} 0$ or $\mathrm{pN} 1$ ), and to evaluate differences in postoperative complications between the initial 50 patients included in this study and the patients in the previous study [9].

The false-negative rate was calculated on a per-patient basis using the following formula: false-negative rate $=[$ false-negative patients $/$ (false-negative patients + truepositive patients)] $\times 100 \%$. The sensitivity of the SN biopsy procedure was also calculated on a per-patient basis using the following formula: [no. of true-positive patients/(no. of true-positive patients + no. of falsenegative patients) $] \times 100 \%$.

\section{Results}

\section{Preoperative imaging}

In 10 of the 55 patients (18.2\%) only unilateral drainage was observed. In the overall group of 55 patients, 147 SNs were identified on the lymphoscintigrams. An additional $65 \mathrm{SNs}$ were identified on SPECT/CT imaging, resulting in a total of 212 SNs (median 4 per patient, IQR 3 - 5) identified on preoperative imaging. Of these 212 preoperatively identified SNs, 55 (26\%) were located outside the ePLND area. These results are specified for each patient group in Table 2 and in Fig. 2.

\section{Intraoperative sentinel node identification}

Of the 55 included patients, 54 were operated upon in a 1-day protocol with a median of $5.04 \mathrm{~h}$ between tracer injection and the start of the operation (IQR $4.33-5.20 \mathrm{~h}$ ). One patient was operated $18.40 \mathrm{~h}$ after tracer injection. The preoperatively

Table 2 SN detection and pathological evaluation

Group

\begin{tabular}{ll}
\hline $\mathrm{SN}+\operatorname{ePLND}(n=50)$ & $\begin{array}{l}\mathrm{SN}+\mathrm{ePLND}, \text { customized } \\
\text { settings }(n=5)\end{array}$
\end{tabular}

\begin{tabular}{|c|c|c|c|}
\hline \multicolumn{4}{|l|}{ SNs detected preoperatively } \\
\hline On lymphoscintigraphy, total & 137 & 10 & 147 \\
\hline On lymphoscintigraphy, per patient, median (IQR) & $2.5(1-4)$ & $2(1-3)$ & $2(1-4)$ \\
\hline On SPECT/CT, total & $4(3-5)$ & $2(1-3.5)$ & $4(3-5)$ \\
\hline On SPECT/CT, per patient, median (IQR) & 201 & 11 & 212 \\
\hline \multicolumn{4}{|l|}{ SNs detected intraoperatively } \\
\hline Total removed & 184 & 14 & 198 \\
\hline No. removed per patient, median (IQR) & $4(2-5)$ & $1(1-5)$ & $4(2-5)$ \\
\hline No. not resected & 36 & 0 & 36 \\
\hline No. additionally resected (as a result of SN cluster formation) & 19 & 3 & 22 \\
\hline \multicolumn{4}{|l|}{ Pathological SN evaluation } \\
\hline No. harvested per patient, median (IQR) & $4(2-6)$ & $1(1-5)$ & $4(2-5)$ \\
\hline Total no. & 226 & 30 & 256 \\
\hline Total no. tumour-positive & 17 & 0 & 17 \\
\hline \multicolumn{4}{|l|}{ Pathological LN evaluation } \\
\hline No. harvested from ePLND specimen per patient, median (range) & $10(8-14)$ & $12(7-22)$ & $10(8-15)$ \\
\hline Total no. & 582 & 69 & 651 \\
\hline Total no. tumour-positive (SNs + LNs) & 41 & 0 & 41 \\
\hline \multicolumn{4}{|l|}{ Pathological SN + LN evaluation (total) } \\
\hline Total no. removed per patient (SN + ePLND), median (IQR) & $15.5(12-20)$ & $18(12-28.5)$ & $16(12-20)$ \\
\hline Total no. harvested $\mathrm{SNs}+\mathrm{LNs}$ & 807 & 99 & 906 \\
\hline Tumour-positive rate, no. (\%) of patients pN1 & $14(28)$ & $0(0)$ & $14(25)$ \\
\hline
\end{tabular}


Fluorescence-based SN identification

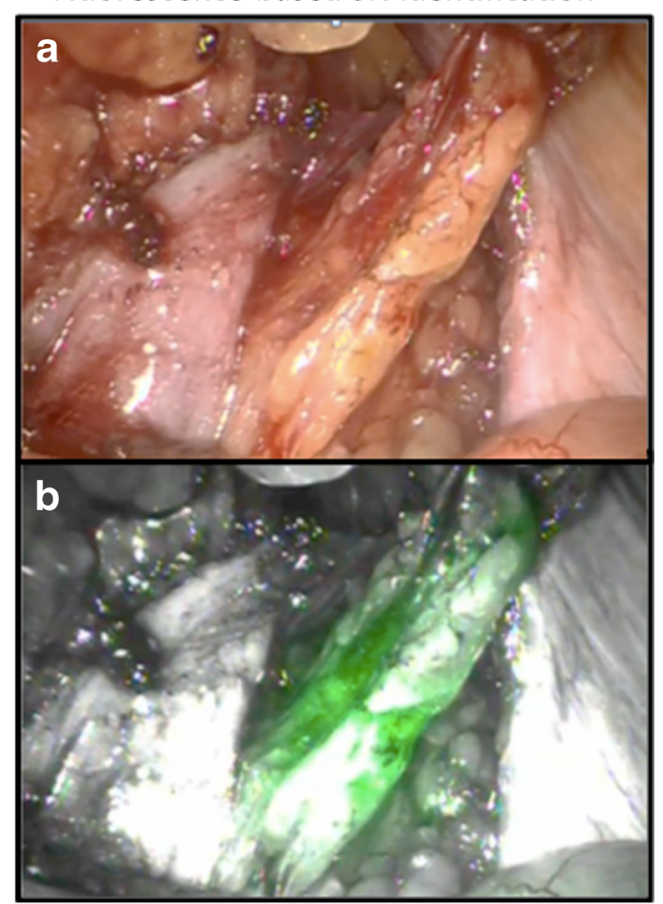

Fig. 2 Locations of SNs and LNs detected intraoperatively. a, b SNs located inside and outside the ePLND area (green in vivo fluorescent SNs, yellow ex vivo identified SNs $n=50$ ). $\mathbf{c}$ SNs that could not be removed (red, $n=55$ ). $\mathbf{d}$, e SNs located in the additionally included five patients inside and outside the ePLND area (green in vivo fluorescent SNs, yellow ex vivo identified SNs). f Location of tumour-positive SNs (black right-sided SNs, grey left-sided SNs). The images were generated using Visible Body software (Argosy Publishing, Newton Upper Falls, MA)

SPECT/CT images were analysed in the operating theatre using an interactive DICOM viewer and provided a virtual starting point for placement of the fluorescence laparoscope in localizing the SNs via fluorescence guidance.

Of the 201 SNs identified in the initial 50 patients on preoperative imaging, $36(17.9 \%)$ were not pursued during the operation. The decision not to resect these SNs was made on the basis of its anatomical location or relationship to close structures, such as presacral and pararectal SNs located close to the rectum or SNs located behind vascular structures such as the internal iliac artery, external iliac artery or vessels in the para-aortal region (Fig. 2c).

As a result of cluster formation, 19 extra SNs were surgically resected based on fluorescence identification. These clustered SNs were seen as one "hotspot" on SPECT, but the CT scan indicated multiple LNs at the location of the hotspot. A total of 148 SNs were removed using intraoperative fluorescence guidance (this was $80.4 \%$ of the 184 surgically resected SNs; Table 2). After removal, ex vivo gamma tracing confirmed excision of these SNs.

The $19.6 \%$ of SNs that could not be resected using fluorescence guidance were resected from the ePLND samples based on the anatomical information provided by the SPECT/CT images and ex vivo gamma tracing and fluorescence imaging. More detailed ex vivo analysis of the ePLND samples allowed fluorescence identification of $97.8 \%$ (180) of the 184 SNs resected and detection of the radioactive signal in $100 \%$ of the SNs.

In the five patients in whom the tunable fluorescence imaging settings were evaluated, as well as the 11 preoperatively identified SNs, 3 more SNs (part of a cluster of SNs) were removed under fluorescence guidance (Table 2). Of the 14 SNs, $11(78.6 \%), 11(78.6 \%)$ and $12(85.7 \%)$ were visualized at $30 \%, 15 \%$ and $0 \%$ white light, respectively (ex vivo, $100 \%$ of the SNs were fluorescent and radioactive). The effect of the light settings on the fluorescence image quality is shown in Fig. 3.

Because the fluorescence laparoscope is an integral part of the da Vinci Si system, the urologist manoeuvred and placed the fluorescence laparoscope independently of the surgical assistant, a feature that had a positive influence on the surgical logistics.

\section{Pathological examination SNs and ePLND specimens}

At pathology, 256 nodes were extracted from the $198 \mathrm{SN}$ specimens surgically excised from the 55 patients, (median 4 per patient, IQR $2-5$ ). From the ePLND specimens a total of $651 \mathrm{LNs}$ were removed, giving a median of $16 \mathrm{LNs}$ per patient. The results are presented in more detail for each patient group in Table 2.

Histopathological examination revealed tumour-positive LNs in $25.4 \%$ of patients (14 of 55 patients, total 41 tumourpositive nodes). In 10 of these $14 \mathrm{pN} 1$ patients $(71.4 \%)$, the SNs were the only tumour-containing nodes. In 3 patients, tumourpositive LNs were also found next to the tumour-positive SNs. The result in one patient was false-negative. In this patient the SN was tumour-negative but a metastasis was detected in a LN from the ePLND tissue specimen, and this LN was not fluorescent or radioactive during surgery. The false-negative rate in this study was therefore $7.1 \%(1 / 14)$. The sensitivity of SN biopsy in this cohort was good: the procedure correctly staged 13 of 14 node-positive patients $(92.9 \%)$. In a previously reported group of patients with similar Briganti scores, we found a tumourpositive node rate of $20 \%$ (8/40 patients) [9]. Compared to the previous study, in the current study, significantly more LNs were removed per patient (median 16 vs. $12 ; p<0.001$ ) and $5.4 \%$ more node-positive patients were identified (14/55 patients; i.e. $25.4 \%$ vs. $20 \%$; $p=0.6$ ). There was no significant difference in the a priori likelihood of $\mathrm{LN}$ metastasis $(p=0.3)$.

\section{Follow-up (complications)}

No significant differences were found in the postoperative complication rates between the patients in the current study and those in the previous study (Table 3) [9]. Changing and 
Fig. 3 Evaluation of adjustable fluorescence settings. SNs identified with various fluorescence settings

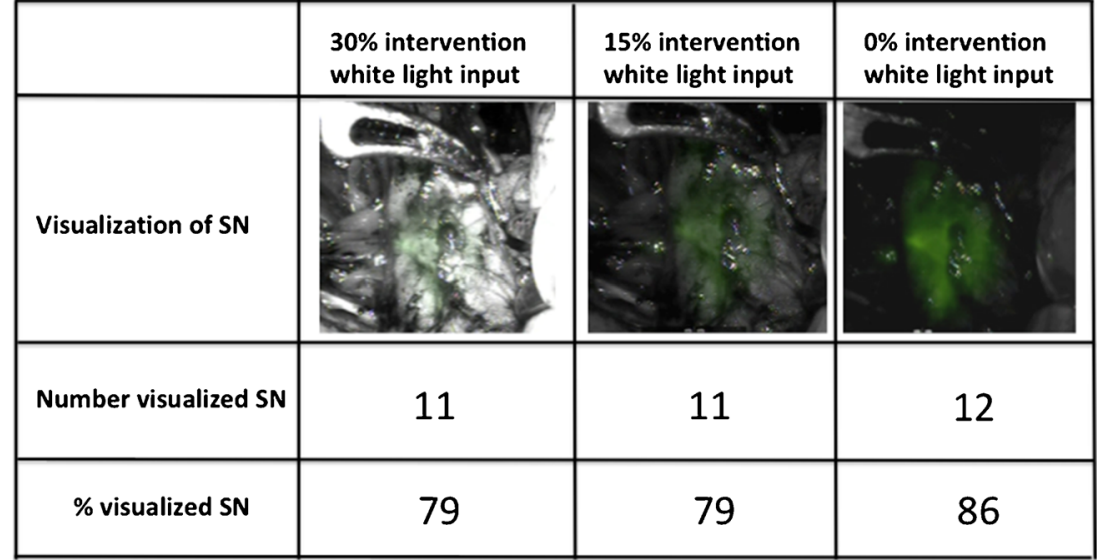

integrating the fluorescence laparoscope did not change the postoperative complication rate.

\section{Discussion}

Integrating molecular imaging and robotic surgery is an important step in the evolution that is taking place in the treatment of prostate cancer. In the current study, using a hybrid (radioactive and fluorescent) SN tracer, findings from nuclear medicine imaging were used to guide the surgical resection using a robot-integrated fluorescence laparoscope. In some patients, it was decided, based on the SPECT/CT information, that some of the preoperatively identified SNs were not eligible for surgical removal. In the remaining patients, the anatomical locations derived from the SPECT/CT images were used to position the fluorescence laparoscope or, when intraoperative fluorescence imaging did not provide accurate $\mathrm{SN}$ identification, to guide resection of the SNs.

Robotic integration of fluorescence imaging gives the urologist more control over the use and positioning of the laparoscope, thus increasing his/her autonomy [21, 22]. To our surprise, using the standard fluorescence imaging settings of the robot-integrated fluorescence laparoscope, integration did not convert into an improved in vivo fluorescence-based SN visualization rate. In fact, the percentage found was lower than the rate we found previously in a setting where the bedside assistant was responsible for placement of an external fluorescence laparoscope [9]. This difference seems to be the result of differences in the camera technology used. However, it may also have been caused by the shorter time spent by the urologist in exploring the area of interest. The last point - saving surgical time to allow accurate fluorescence identification seems to be in line with our previous finding [9]. Nevertheless, the fluorescence imaging miss-rate of

Table 3 Complication rate

\begin{tabular}{|c|c|c|c|c|}
\hline Complication & $\begin{array}{l}\text { Clavien-Dindo } \\
\text { grade }\end{array}$ & $\begin{array}{l}\text { This study, group } \\
\text { SN }+ \text { ePLND }(n=50)\end{array}$ & $\begin{array}{l}\text { KleinJan et al. [9] } \\
(n=40)\end{array}$ & $\begin{array}{l}\text { Total } \\
(\mathrm{n}=90)\end{array}$ \\
\hline Lymphocele & IIIa & $1(2)$ & $2(5)$ & $3(3)$ \\
\hline Urinary tract infection & II & $2(4)$ & $2(5)$ & $4(4)$ \\
\hline Postoperative bowel obstruction & II & $1(2)$ & $1(3)$ & $2(2)$ \\
\hline $\begin{array}{l}\text { Micturition obstruction } \\
\text { (Sachse ureterotomy) }\end{array}$ & $\mathrm{IIIb}$ & 0 & $1(3)$ & $1(1)$ \\
\hline $\begin{array}{l}\text { Hematoma of the ventral } \\
\text { abdominal wall }\end{array}$ & I & $1(2)$ & 0 & $2(9)$ \\
\hline $\begin{array}{l}\text { Postoperative infected abdominal } \\
\text { haematoma }\end{array}$ & II & $1(2)$ & $1(3)$ & $2(9)$ \\
\hline Leakage of anastomosis & $\mathrm{I} / \mathrm{III}$ & $2(4)$ & $0(0)$ & $2(2)$ \\
\hline Postoperative wound infection & II & 0 & $1(3)$ & $1(1)$ \\
\hline Epididymitis & II & 0 & $1(3)$ & $1(1)$ \\
\hline Hydronephrosis & IIIa & 0 & $1(3)$ & $1(1)$ \\
\hline Deep venous thrombosis & II & $1(2)$ & 0 & $1(1)$ \\
\hline Total & & $9(18)$ & $10(25)^{*}$ & $19(21)$ \\
\hline
\end{tabular}

Values are number $(\%)$ of patients

$* p=0.4$ 
$15-20 \%$ (in the 55 patients) means that with fluorescence guidance alone a large number of SNs would have been missed. Due to the hybrid nature of the tracer used, the radioactive signature could be used to compensate for this shortcoming and to identify the additional SNs. Rather than using cumbersome laparoscopic gamma tracing, which again has to be performed by the bedside assistant, we used a more time effective combination of ex vivo gamma tracing and SPECT/ CT images to guide resection of the residual SNs. Next to the radioactive signatures, the fluorescent signatures we verified ex vivo considerably increased the fluorescence-based SN detection rate $(>97 \%)$. This finding emphasizes that the tissue attenuation encountered in the in vivo situation limits the success of the fluorescence guidance approach, rather than the presence of tracer in the SNs. It also indicates that the backup from SPECT/CT is of the utmost importance. In an ongoing randomized controlled study we are evaluating these aspects in more detail.

In the five patients in whom the fluorescence settings could be adjusted, decreasing the percentage of white light, and thus decreasing the level of anatomical detail in the fluorescence image, helped improve the detection rate to $85.7 \%$. This improvement seems to contradict our previous findings. With a different fluorescence laparoscope, the introduction of whitelight anatomical background information positively influenced the intraoperative fluorescence-based identification rate [9]. In this previous set-up we did not determine if the application of flexible white-light settings could improve fluorescence detection rates even further. The increased time used for the fluorescence detection at different light settings could also have played influenced the detection rate. Based on the current findings, an adjustable illuminator, wherein white light intensity can be tailored, may most flexibly accommodate the urologist's needs.

One may reason that increasing the proportion of tumour-positive LNs resected will increase the likelihood of the patient's recovery from the disease. In this sense, the $5.4 \%$ greater rate of detection of tumour-positive LNs and the higher sensitivity than in our previous study (92.9\% vs. $75.0 \%$ \%) [9] can be considered valuable. However, we cannot fully attribute this result to the surgical procedure. The tumour-positive $\mathrm{LN}$ detection rate may partly also be affected by the increased number of resected SNs and the pathological examination of the specimens. SNs have a higher chance of containing (micro)metastases and thus have a prognostic value [23] and as such receive more careful pathological evaluation including immunohistochemistry and cutting deeper levels than LNs out of a dissection template [24-27].

A crucial aspect of the clinical introduction of a new technology is the evaluation of the influence the technology will have on clinical outcome. For fluorescence guidance technologies such information is limited [14].
While one may reason that the SN biopsy procedure increases the chance of postoperative complications, the complication rate we observed was lower than in our previous study $(p=0.4)$ [9]. In both studies this result may in part be a consequence of using SPECT/CT planning to guide the procedure away from SNs located in areas that may be associated with postoperative complications.

The extensive introduction of new surgical technologies means that a need has been established for platforms that allow the integration of numerous imaging findings into the surgical workflow. In our view the da Vinci robot platform may act as such a linking technology. For example, robotic surgical goggles have been used to introduce complementary intraoperative and preoperative imaging findings as well as virtual/augmented reality displays $[12,28]$. Since in the current study it was not possible to interactively examine the DICOM SPECT/CT images in Tilepro ${ }^{\circledR}$ during the operation, the urologist had to leave the console to examine them on a separate DICOM viewing station. However, in another study we have already provided a proof-of-concept that directly linking SPECT/CT imaging information and fluorescence imaging during robot-assisted procedures may provide the next step in surgical guidance [29].

The hybrid tracer concept illustrated in this study and our previous prostate cancer-related studies $[9,10,30]$ provides a valuable extension of routine SN identification. A similar hybrid concept holds promise for (tumour-)targeted tracers and may benefit from the set up we have applied for SN identification. One example of a target that holds promise for image-guided resection is prostate-specific membrane antigen (PSMA), which is already routinely used in PET/ CT-based diagnostics $\left({ }^{68} \mathrm{Ga}\right.$-PSMA-HBED-CC) and has been successfully used for radioguided resection $\left({ }^{111}\right.$ In-PSMA I\&T) [31]. Theoretically, a hybrid PSMA derivative [32] would extend these efforts to accommodate (robotintegrated) hybrid surgical guidance towards the primary tumour margins, lymphatic metastases, and possibly even other distant metastases.

\section{Conclusion}

Hybrid tracers help to integrate nuclear medicine and fluorescence-guided robotic surgery, but the use of a robotintegrated fluorescence laparoscope did not improve fluorescence-based SN identification. Hence, 3D preoperative imaging information from nuclear medicine remains crucial for (virtual) planning of complex surgical resections of multifocal lesions. Further technical refinement of robot-integrated guidance modalities in surgical procedures should improve the relationship between preoperative and intraoperative imaging findings. 
Acknowledgments We gratefully acknowledge the entire surgical staff, and the Department of Pathology (and specifically the CF-MPB), of The Netherlands Cancer Institute - Antoni van Leeuwenhoek Hospital, for their assistance.

\section{Compliance with ethical standards}

Funding This work was partially supported by a Dutch Cancer Society translational research award (grant no. PGF 2009-4344), a NWO-STWVIDI grant (grant no. STW BGT11272), and a grant from the European Research Council under the European Union's Seventh Framework Programme (FP7/2007-2013, grant no. 2012-306890).

\section{Conflicts of interest None.}

Research involving human participants All procedures performed in studies involving human participants were in accordance with the ethical standards of our institution and with the principles of the 1964 Declaration of Helsinki and its later amendments or comparable ethical standards.

Informed consent Informed consent was obtained from all individual participants included in the study.

Open Access This article is distributed under the terms of the Creative Commons Attribution 4.0 International License (http:// creativecommons.org/licenses/by/4.0/), which permits unrestricted use, distribution, and reproduction in any medium, provided you give appropriate credit to the original author(s) and the source, provide a link to the Creative Commons license, and indicate if changes were made.

\section{References}

1. Vermeeren L, van der Ploeg IMC, Olmos RA V, Meinhardt W, Klop WMC, Kroon BBR, et al. SPECT/CT for preoperative sentinel node localization. J Surg Oncol. 2010;101(2): 184-90.

2. Zaknun JJ, Giammarile F, Olmos RAV, Vidal-Sicart S, Mariani G. Changing paradigms in radioguided surgery and intraoperative imaging: the GOSTT concept. Eur J Nucl Med Mol Imaging. 2012;39(1):1-3.

3. Mariani G, Erba P, Manca G, Villa G, Gipponi M, Boni G, et al. Radioguided sentinel lymph node biopsy in patients with malignant cutaneous melanoma: the nuclear medicine contribution. J Surg Oncol. 2004;85(3):141-51.

4. Mariani G, Erba P, Villa G, Gipponi M, Manca G, Boni G, et al. Lymphoscintigraphic and intraoperative detection of the sentinel lymph node in breast cancer patients: the nuclear medicine perspective. J Surg Oncol. 2004;85(3):112-22.

5. Van der Zee AGJ, Oonk MH, De Hullu JA, Ansink AC, Vergote I, Verheijen RH, et al. Sentinel node dissection is safe in the treatment of early-stage vulvar cancer. J Clin Oncol. 2008;26(6):884-9.

6. Buckle T, Brouwer OR, Valdés Olmos RA, van der Poel HG, van Leeuwen FWB. Relationship between intraprostatic tracer deposits and sentinel lymph node mapping in prostate cancer patients. J Nucl Med. 2012;53(7):1026-33.

7. Holl G, Dorn R, Wengenmair H, Weckermann D, Sciuk J. Validation of sentinel lymph node dissection in prostate cancer: experience in more than 2,000 patients. Eur J Nucl Med Mol Imaging. 2009;36(9):1377-82.
8. Acar C, Kleinjan GH, van den Berg NS, Wit EM, van Leeuwen FW, van der Poel HG. Advances in sentinel node dissection in prostate cancer from a technical perspective. Int J Urol. 2015;22(10):898-909. doi:10.1111/iju.12863.

9. KleinJan GH, van den Berg NS, Brouwer OR, de Jong J, Acar C, Wit EM, et al. Optimisation of fluorescence guidance during robotassisted laparoscopic sentinel node biopsy for prostate cancer. Eur Urol. 2014;66(6):991-8.

10. van der Poel HG, Buckle T, Brouwer OR, Valdés Olmos RA, van Leeuwen FWB. Intraoperative laparoscopic fluorescence guidance to the sentinel lymph node in prostate cancer patients: clinical proof of concept of an integrated functional imaging approach using a multimodal tracer. Eur Urol. 2011;60(4):826-33.

11. Manny TB, Patel M, Hemal AK. Fluorescence-enhanced robotic radical prostatectomy using real-time lymphangiography and tissue marking with percutaneous injection of unconjugated indocyanine green: the initial clinical experience in 50 patients. Eur Urol. 2014;65(6):1162-8.

12. Volonté F, Buchs NC, Pugin F, Spaltenstein J, Schiltz B, Jung M, et al. Augmented reality to the rescue of the minimally invasive surgeon. The usefulness of the interposition of stereoscopic images in the Da Vinci ${ }^{\mathrm{TM}}$ robotic console. Int J Med Robot. 2013;9(3):e34-8.

13. Jewell EL, Huang JJ, Abu-Rustum NR, Gardner GJ, Brown CL, Sonoda $\mathrm{Y}$, et al. Detection of sentinel lymph nodes in minimally invasive surgery using indocyanine green and near-infrared fluorescence imaging for uterine and cervical malignancies. Gynecol Oncol. 2014;133(2):274-7.

14. van Leeuwen FW, Hardwick JC, van Erkel AR. Luminescencebased imaging approaches in the field of interventional molecular imaging. Radiology. 2015;276(1):12-29.

15. van den Berg NS, Brouwer OR, Schaafsma BE, Mathéron HM, Klop WM, Balm AJ, et al. Multimodal surgical guidance during sentinel node biopsy for melanoma: combined gamma tracing and fluorescence imaging of the sentinel node through use of the hybrid tracer indocyanine green-(99m)Tc-nanocolloid. Radiology. 2015;275(2):521-9.

16. Brouwer OR, van den Berg NS, Mathéron HM, van der Poel HG, van Rhijn BW, Bex A, et al. A hybrid radioactive and fluorescent tracer for sentinel node biopsy in penile carcinoma as a potential replacement for blue dye. Eur Urol. 2014;65(3):600-9.

17. Mathéron HM, van den Berg NS, Brouwer OR, Kleinjan GH, van Driel WJ, Trum JW, et al. Multimodal surgical guidance towards the sentinel node in vulvar cancer. Gynecol Oncol. 2013;131(3): $720-5$.

18. Briganti A, Chun FK-H, Salonia A, Zanni G, Scattoni V, Valiquette L, et al. Validation of a nomogram predicting the probability of lymph node invasion among patients undergoing radical prostatectomy and an extended pelvic lymphadenectomy. Eur Urol. 2006;49(6):1019-26; discussion 1026-7.

19. Heidenreich A, Bastian PJ, Bellmunt J, Bolla M, Joniau S, van der Kwast T, et al. EAU guidelines on prostate cancer. Part 1: screening, diagnosis, and local treatment with curative intent - update 2013. Eur Urol. 2014;65(1):124-37.

20. Clavien PA, Barkun J, de Oliveira ML, Vauthey JN, Dindo D, Schulick RD, et al. The Clavien-Dindo classification of surgical complications: five-year experience. Ann Surg. 2009;250(2):18796.

21. Van Oosterom MN, Simon H, Laurent M, Welling MM, Van der Poel HG, Van den Berg NS, et al. Revolutionizing (robot-assisted) laparoscopic gamma tracing using a drop-in gamma probe technology. Am J Nucl Med Mol Imaging. 2015. In press.

22. Fuerst B, Sprung J, Pinto F, Frisch B, Wendler T, Simon H, et al. First robotic SPECT for minimally invasive sentinel lymph node mapping. IEEE Trans Med Imaging. 2015;PP(99):1. doi:10.1109/ TMI.2015.2498125 
23. Holtkamp LH, Wang S, Wilmott JS, Madore J, Vilain R, Thompson $\mathrm{JF}$, et al. Detailed pathological examination of completion node dissection specimens and outcome in melanoma patients with minimal $(<0.1 \mathrm{~mm})$ sentinel lymph node metastases. Ann Surg Oncol. 2015;22(9):2972-7.

24. Gofrit ON, Zorn KC, Steinberg GD, Zagaja GP, Shalhav AL. The Will Rogers phenomenon in urological oncology. J Urol. 2008;179(1):28-33.

25. Staník M, Capák I, Macík D, Vašina J, Lžičařová E, Jarkovský J, et al. Sentinel lymph node dissection combined with meticulous histology increases the detection rate of nodal metastases in prostate cancer. Int Urol Nephrol. 2014;46(8):1543-9.

26. Wawroschek F, Wagner T, Hamm M, Weckermann D, Vogt H, Märkl B, et al. The influence of serial sections, immunohistochemistry, and extension of pelvic lymph node dissection on the lymph node status in clinically localized prostate cancer. Eur Urol. 2003;43(2):132-7.

27. Fukuda M, Egawa M, Imao T, Takashima H, Yokoyama K, Namiki $\mathrm{M}$. Detection of sentinel node micrometastasis by step section and immunohistochemistry in patients with prostate cancer. J Urol. 2007;177(4):1313-7; discussion 1317.
28. Greco F, Cadeddu JA, Gill IS, Kaouk JH, Remzi M, Thompson RH, et al. Current perspectives in the use of molecular imaging to target surgical treatments for genitourinary cancers. Eur Urol. 2014;65(5): 947-64.

29. Brouwer OR, Buckle T, Bunschoten A, Kuil J, Vahrmeijer AL, Wendler $\mathrm{T}$, et al. Image navigation as a means to expand the boundaries of fluorescence-guided surgery. Phys Med Biol. 2012;57(10): 3123-36.

30. van den Berg NS, Valdés-Olmos RA, van der Poel HG, van Leeuwen FWB. Sentinel lymph node biopsy for prostate cancer: a hybrid approach. J Nucl Med. 2013;54(4):493-6.

31. Maurer T, Weirich G, Schottelius M, Weineisen M, Frisch B, Okur A, et al. Prostate-specific membrane antigen-radioguided surgery for metastatic lymph nodes in prostate cancer. Eur Urol. 2015;68(3):530-4.

32. Banerjee SR, Pullambhatla M, Byun Y, Nimmagadda S, Foss CA, Green G, et al. Sequential SPECT and optical imaging of experimental models of prostate cancer with a dual modality inhibitor of the prostate-specific membrane antigen. Angew Chem Int Ed Engl. 2011;50(39):9167-70. 